\title{
Corrigendum to "Orlicz-Lorentz Spaces and their Composition Operators" [Proyecciones (Antofagasta). 34, (2015) No 1, pp. 85-105.]
}

\author{
René Erlin Castillo \\ Universidad Nacional de Colombia, Departamento de Matemáticas \\ recastillo@unal.edu.co \\ Héctor Camilo Chaparro \\ Universidad Nacional de Colombia, Departamento de Matemáticas \\ hcchaparrog@unal.edu.co \\ Bogotá, Colombia \\ Julio César Ramos Fernández \\ Universidad de Oriente, Departamento de Matemáticas \\ 6101 Cumaná, Estado Sucre, Venezuela \\ jcramos@udo.edu.ve
}

\begin{abstract}
After publication of the above article in Proyecciones (Antofagasta). Volume 34, (2015) No 1, pp. 85-105, it has come to the attention of the authors that an extra condition was missing in Theorem 3.2 .
\end{abstract}

\section{Introduction}

Recently it was called to our attention by professor Lech Maligranda that Theorem 3.2 in Orlicz-Lorentz Spaces and their Composition Operators (see [1]) fails to be true when the weight $w \equiv 1$, it is necessary to additionally suppose the $\Delta_{2}$-condition.

*2010 Mathematics Subject Classification: Primary 47B33, 47B38, Secondary 46E30 
Now, in order that everything will be in a good shape, we rewrite Theorem 3.2 as follows

Theorem 1.1. Let $T: X \rightarrow X$ be a non-singular measurable transformation. If $C_{T}$ induced by $T$ is bounded, then there exists $M \geq 1$ such that $\nu\left(T^{-1}(A) \leq M \nu(A)\right)$.

In addition, if $\varphi$ satisfies the $\Delta_{2}$-condition for all $t>0$ and $\nu\left(T^{-1}(A)\right) \leq M \nu(A)$ for $A \in \mathcal{A}$, then $C_{T}$ is bounded. Moreover,

$$
\left\|C_{T} f\right\|=\sup _{0<\nu(A)<\infty}\left(\frac{\nu\left(T^{-1}(A)\right)}{\nu(A)}\right) .
$$

Proof. We empathize that the fist part of the proof of this Theorem goes the same as in the proof of Theorem 3.2 in [1] and it holds without the $\Delta_{2}$-condition. Conversely, if $\nu\left(T^{-1}(A)\right) \leq M \nu(A)$ holds for all $A \in \mathcal{A}$, then

$$
\begin{aligned}
& \int_{0}^{\mu\left(T^{-1}(A)\right)} w(t) d t \leq M \int_{0}^{\mu(A)} w(t) d t \\
\Rightarrow & \int_{0}^{\infty} \chi_{\left(0, \mu\left(T^{-1}(A)\right)\right)}(t) w(t) d t \leq M \int_{0}^{\infty} \chi_{(0, \mu(A))}(t) w(t) d t \\
\Rightarrow & \int_{0}^{\infty} \chi_{T^{-1}(A)}^{*}(t) w(t) d t \leq M \int_{0}^{\infty} \chi_{A}^{*}(t) w(t) d t \\
\Rightarrow & \int_{0}^{\infty}\left(\chi_{E} \circ T\right)^{*}(t) w(t) d t \leq M \int_{0}^{\infty} \chi_{A}^{*}(t) w(t) d t \\
\Rightarrow & \int_{0}^{\infty}(f \circ T)^{*}(t) w(t) d t \leq M \int_{0}^{\infty} f^{*}(t) w(t) d t
\end{aligned}
$$

Therefore

$$
(f \circ T)^{*}(t) \leq M f^{*}(t) \quad \text { a.e. }
$$

Since $\varphi$ satisfies the $\Delta_{2}$-condition for all $t>0$, it follows that

$$
k=\sup _{t>0} \frac{\varphi(\alpha t)}{\varphi(t)}<\infty
$$

and thus

$$
\varphi(\alpha t) \leq k \varphi(t)
$$

then, with $k=1 / M$, we have

$$
\begin{aligned}
\int_{0}^{\infty} \varphi\left(\frac{(f \circ T)^{*}(t)}{M\|f\|_{\varphi, w}}\right) w(t) d t & \leq \frac{1}{M} \int_{0}^{\infty} \varphi\left(\frac{f^{*}(t)}{\|f\|_{\varphi, w}}\right) w(t) d t \\
& \leq \int_{0}^{\infty} \varphi\left(\frac{f^{*}(t)}{\|f\|_{\varphi, w}}\right) w(t) d t \leq 1 .
\end{aligned}
$$


Finally

$$
\|f \circ T\|_{\varphi, w} \leq M\|f\|_{\varphi, w},
$$

that is

$$
\left\|C_{T} f\right\|_{\varphi, w} \leq M\|f\|_{\varphi, w} .
$$

On the one hand, let us prove (1). Indeed, let

$$
N=\sup _{0<\nu(A)<\infty}\left(\frac{\nu\left(T^{-1}(A)\right)}{\nu(A)}\right),
$$

then

$$
\nu\left(T^{-1}(A)\right) \leq N \nu(A)
$$

and thus

$$
\left\|C_{T} f\right\|_{\varphi, w} \leq N\|f\|_{\varphi, w}, \quad \forall f \in L_{\varphi, w}
$$

hence

$$
\frac{\left\|C_{T} f\right\|_{\varphi, w}}{\|f\|_{\varphi, w}} \leq N, \quad \text { for all } 0 \neq f \in L_{\varphi, w}
$$

Therefore

$$
\begin{aligned}
\left\|C_{T}\right\| & =\sup _{f \neq 0} \frac{\left\|C_{T}(f)\right\|_{\varphi, w}}{\|f\|_{\varphi, w}} \\
& <N=\sup _{0<\nu(A)<\infty}\left(\frac{\nu\left(T^{-1}(A)\right)}{\nu(A)}\right) .
\end{aligned}
$$

That is

$$
\left\|C_{T}\right\| \leq \sup _{0<\nu(A)<\infty}\left(\frac{\nu\left(T^{-1}(A)\right)}{\nu(A)}\right) .
$$

On the other hand, let us consider

$$
M=\left\|C_{T}\right\|=\sup _{f \neq 0} \frac{\left\|C_{T}(f)\right\|_{\varphi, w}}{\|f\|_{\varphi, w}}
$$

then

$$
\frac{\left\|C_{T}(f)\right\|_{\varphi, w}}{\|f\|_{\varphi, w}} \leq M \quad \forall 0 \neq f \in L_{\varphi, w} .
$$

In particular, if $f=\chi_{A}$ such that $0<\mu(A)<\infty, A \in \mathcal{A}$, then

$$
\frac{\left\|C_{T}\left(\chi_{A}\right)\right\|_{\varphi, w}}{\left\|\chi_{A}\right\|_{\varphi, w}}=\left(\frac{\nu\left(T^{-1}(A)\right)}{\nu(A)}\right) \leq M,
$$


therefore

$$
\sup _{0<\nu(A)<\infty}\left(\frac{\nu\left(T^{-1}(A)\right)}{\nu(A)}\right) \leq M=\left\|C_{T}\right\| .
$$

Combining (2) and (3) we have

$$
\left\|C_{T}\right\|=\sup _{0<\nu(A)<\infty}\left(\frac{\nu\left(T^{-1}(A)\right)}{\nu(A)}\right) .
$$

Remark 1.2. We like to point it out that when the weight $w(t)=1$ we obtain the characterization for boundedness of the composition operator in Orlicz space (see Theorem 2.2. in [2]).

\section{Acknowledgement}

The authors wish to express their gratitude to professor Lech Maligranda for called our attention on the missing condition in Theorem 3.2 .

\section{References}

[1] R.E. Castillo, H. C. Chaparro and J. C. Ramos Fernández. OrliczLorentz spaces and their Composition Operators. Proyecciones (Antofagasta), 34(1), 85-105.

[2] Y. Cui, H. Hudzik, Romesh Kumar and L. Maligranda, Composition operators in Orlicz spaces, J. Austral. Math. Soc., Vol. 76(2) (2004), 189-206. 